\title{
Magnetic Moment of Zigzag CuO Nanotubes at Different Temperature and Size: Ab-Initio Study
}

\author{
Paudel $\mathrm{S}^{1,2}$, Yadav $\mathrm{TP}^{3,4}$, Srivastava $\mathrm{A}^{1}$ and Kaphle $\mathrm{GC}^{\star 3,4,5}$ \\ ${ }^{1}$ Advanced Materials Research Group, Computational Nanoscience and Technology Laboratory, ABV-Indian \\ Institute of Information Technology and Management, Gwalior, M.P, India \\ ${ }^{2}$ Department of Physics, Tribhuvan University, Patan Multiple Campus, Patandhoka, Lalitpur, Nepal \\ ${ }^{3}$ Central Department of Physics, Tribhuvan University, Kirtipur, Kathmandu, Nepal \\ ${ }^{4}$ Hydra Research and Policy Center Kathamndu, Nepal \\ ${ }^{5}$ Condensed Matter Physics Research Center, Butwal, Rupandehi, Nepal
}

${ }^{*}$ Corresponding author: Kaphle GC, Central Department of Physics, Tribhuvan University, Kirtipur, Kathmandu, Nepal, Tel: +9779849000975, E-mail: gck223@gmail.com

Citation: Paudel S, Yadav TP, Srivastava A, Kaphle GC (2017) Magnetic Moment of Zigzag CuO Nanotubes at Different Temperature and Size: Ab-initio study. J Mat Sci Nanotechol 6(1): 102

Received Date: October 09, 2017 Accepted Date: January 02, 2018 Published Date: January 05, 2018

\begin{abstract}
Temperature and size dependence magnetic moment of zigzag $(n, 0)(n=4,6,8,10)$ copper oxide nanotubes $(\mathrm{CuO} N T s)$ have been computed by using a standard density functional theory. The computational work carried out by employing spin polarized generalized gradient approximation with revised Perdew Burke Ernzerhoff type parameterization along ab-initio approach. Bond length, binding energy and total magnetic moment have been analyzed and found that the bond length decreases at first and become saturated with increasing size of the nanotube. Highest binding energy of $(10,0)$ confirms this as the most stable amongst all the NTs taken into consideration. The observed electronic properties of considered $\mathrm{CuO}$ NTs, confirms the metallic nature. The size dependence magnetic moment at room temperature is decreases around up to $(8,0)$ beyond that magnetic moments found to be increased with the diameter of the nanotubes. The computed temperature dependence's magnetic moment of NTs first increase up to room temperature (300K) and then decreases for all NTs except $(8,0)$.
\end{abstract}

Keywords: Density Functional Theory (DFT); Bond length, Binding energy; Magnetic moment; CuO Nanotube

\section{Introduction}

In the past few years' investigation in the magnetic properties with different aspect in nano structure have been increased rapidly because of their technological importance, lots of attractive properties like, high surface-volume ratio, electronic, optical and magnetic properties as well as for understanding the physics involved in their many unusual properties comparing with the bulk [1-5]. Since, the discovery of the high temperature superconductor in copper oxide peroviskit and its unique electric and magnetic properties, Copper oxide $(\mathrm{CuO})$ got more attention and made more interesting candidate for research [6-8]. Our recent ab-intio investigation on magnetism in zigzag and armchair $\mathrm{CuO}$ nanotubes shows remarkable magnetic properties at different diameter [9]. So, it is wrathful to investigate the temperature and size dependent magnetic properties in zigzag CuO nano tubes as a function of temperature.

As a bulk $\mathrm{CuO}$ is a p-type semiconductor with band gap of 1.1-1.9 eV, unlike other transition metal oxide semiconductor, it have monoclinic structure and possess versatile band structure, optical and magnetic properties [8,10,11].

Our work focuses on the stability analysis of zigzag $\mathrm{CuO}$ nanotube in terms of its binding energy, diameter along with temperature dependence of magnetic properties. Organization of this article is as follows, next section discusses the method and computational details followed by result and discussion section and conclusion of the work is presented before the references.

\section{Method and Computational Detail}

Computational calculation has been done by Density Functional Theory (DFT), based on ab-initio code named Atomisitix Toolkit (ATK-VNL) with non-equilibrium Green Function (NEGF) [12-15]. ATK-VNL is a further development of TransSIESTA-C and is based on the methodology, models, algorithms developed in academic code TransSIESTA and in part McDCal employing localized 
in SIESTA [16-18]. Spin Generalized Gradient Approximation with revised Perdew Burke Eenzerhoff used for exchange correlation energy $[19,20]$. Valence electrons were described by localized pseudo atomic orbital's (PAOs), with double $\zeta$ double polarized basis set. Mesh cut-off 75 Hartee is applied in entire calculation, with K-point sampling of $1 \times 1 \times 20$ and maximum force tolerance set at $0.05 \mathrm{eV} / \mathrm{A}[21]$. Calculation was began by minimization energy, optimized geometry and further. Minimization of energy gives us stable bond length between $\mathrm{Cu}$ and $\mathrm{O}$. The stable bond length is used to creates the $\mathrm{CuO}$ zigzag nanotubes using ATK-VNL tool.

\section{Results and Discussion}

\section{Stability and Structural Analysis}

The current work carries the analysis of diameter dependence of structural stability and electronic properties. The diameter and temperature dependence magnetic properties of zigzag $\mathrm{CuO}$ nanotubes also studied shown in Figure 1.The calculated magnetic properties and structure parameter have been listed in Table 1. From Table 1 for the larger diameter nanotubes, the number of atom and Binding energy $\left(\mathrm{E}_{\mathrm{b}}\right)$ increases, whereas bond length between $\mathrm{Cu}-\mathrm{O}$ is first increased and then become saturated. The nanotube of chirality $(4,0)$ is found to be stable at bond length $(\mathrm{Cu}-\mathrm{O})$ of $1.95 \AA$, where chirality $(10,0)$ is stable at $1.90 \AA$. The buckle appeared on the optimized structure of nanotube decreases with increasing the diameter as shown in Figure 1. This is mainly due to well known effect of reduction of bond strain on the curvature surface with increase in the diameter of nanotube [9].

Binding energy of the NTs are calculated through following relation;

$$
E_{b}=\frac{\left[\mathrm{N}^{*} \mathrm{E}_{T}\left(\mathrm{Cu}_{\text {iso }}+M * \mathrm{E}_{T}\left(\mathrm{O}_{\text {iso }}\right)-\mathrm{E}_{T}(\mathrm{CuONT})\right]\right.}{N+M}
$$

Where $\mathrm{N}=$ number of copper atoms present in SWNT

$\mathrm{M}=$ number of Oxygen atoms present in SWNT

$\mathrm{E}_{\mathrm{T}}=$ Total energy of $\mathrm{Cu}, \mathrm{O}$ and $\mathrm{CuO}$ SWNTs respectively

Similarly, the binding energy is calculated through equation 1 and listed in Table 1, which concludes that the binding energy increases significantly with diameter of zigzag nano tubes for small value of diameter and after certain increase in the diameter it increase very slowly: this character of binding energy arise due to reduction in the strain.

\begin{tabular}{|c|c|c|c|c|c|}
\hline $\begin{array}{c}\text { Chirality } \\
(\mathbf{n}, \mathbf{0})\end{array}$ & $\begin{array}{c}\text { No. of } \\
\text { atom N }\end{array}$ & $\begin{array}{c}\text { Bond length } \\
(\mathbf{C u}-\mathbf{O}) \AA\end{array}$ & $\begin{array}{c}\text { Diameter } \\
\AA\end{array}$ & $\begin{array}{c}\mathbf{E}_{\mathrm{B}} \\
(\mathbf{e V})\end{array}$ & $\begin{array}{c}\boldsymbol{\mu} \text { total }(\boldsymbol{\mu B} / \\
\text { atom })\end{array}$ \\
\hline$(4,0)$ & 16 & 1.95 & 4.30 & 4.73 & 0.453 \\
\hline$(6,0)$ & 24 & 1.90 & 6.29 & 4.81 & 0.314 \\
\hline$(8,0)$ & 32 & 1.90 & 8.39 & 4.84 & 0.191 \\
\hline$(10,0)$ & 40 & 1.90 & 10.46 & 4.85 & 0.230 \\
\hline
\end{tabular}

Table 1: Bond length, Binding energy, Diameter and Total Magnetic Moment at room temperature of $\mathrm{CuO}$ SWNTs

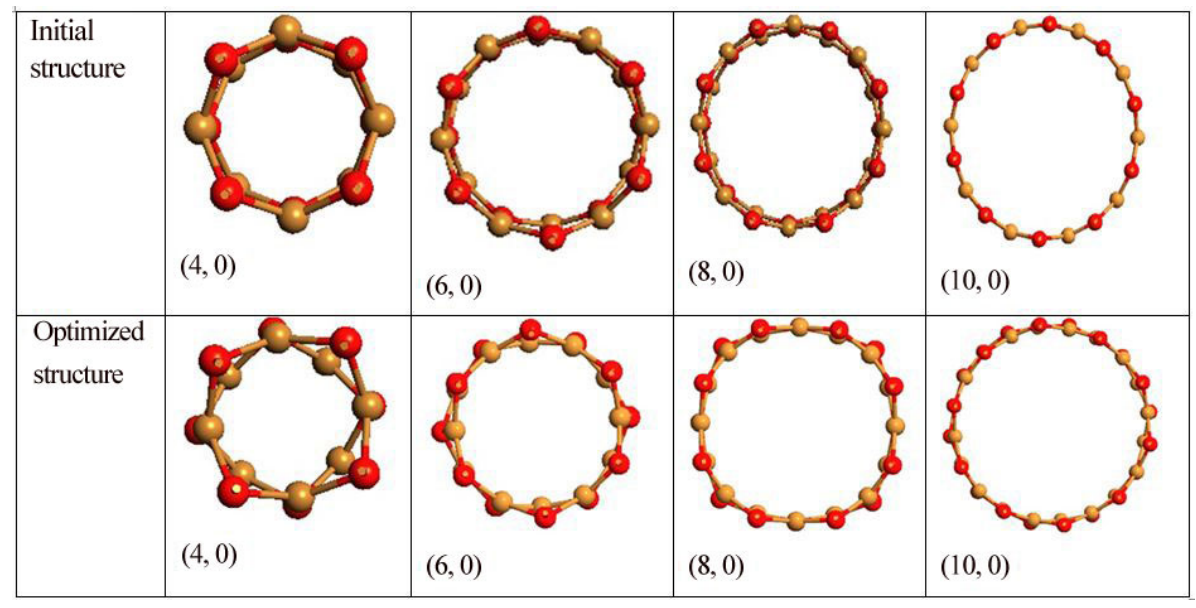

Figure 1: (color online) Initial and optimized geometries of CuO NTs

\section{Electronic Property}

Band structure of $\mathrm{CuO}$ zigzag nanotubes with majority and minority spin at room temperature are plotted in Figure 2 . Where black lines indicate the majority spin and red lines indicates the minority spin. Our work shows that zigzag CuO nanotubes are metallic in nature, but the bulk $\mathrm{CuO}$ is a p-type semiconductor. This is responsible due to hybridization between $\mathrm{O}-2 \mathrm{p}$ and $\mathrm{Cu}-3 \mathrm{~d}$ states near the Fermi level. This nature observed here in our case is in line with that reported earlier [22,23]. 
At different diameter, and at room temperature, the density of state (DOS) of zigzag CuO nanotubes have also been observed using spin polarization DFT calculation and plotted at Figure 3. The peaks appear near the Fermi levels in valance band are because of $\mathrm{Cu}-3 \mathrm{~d}$ and $\mathrm{O}-2 \mathrm{p}$ states, while the peak in conduction band is due to $4 \mathrm{~s}$ state of $\mathrm{Cu}$. The finite contribution of $\mathrm{O}$ atom to the density of state at Fermi level might be a reason for the metallic nature of nanotube, besides the hybridization on of $\mathrm{Cu}-3 \mathrm{~d}$ and $\mathrm{O}-2 \mathrm{p}$ states.

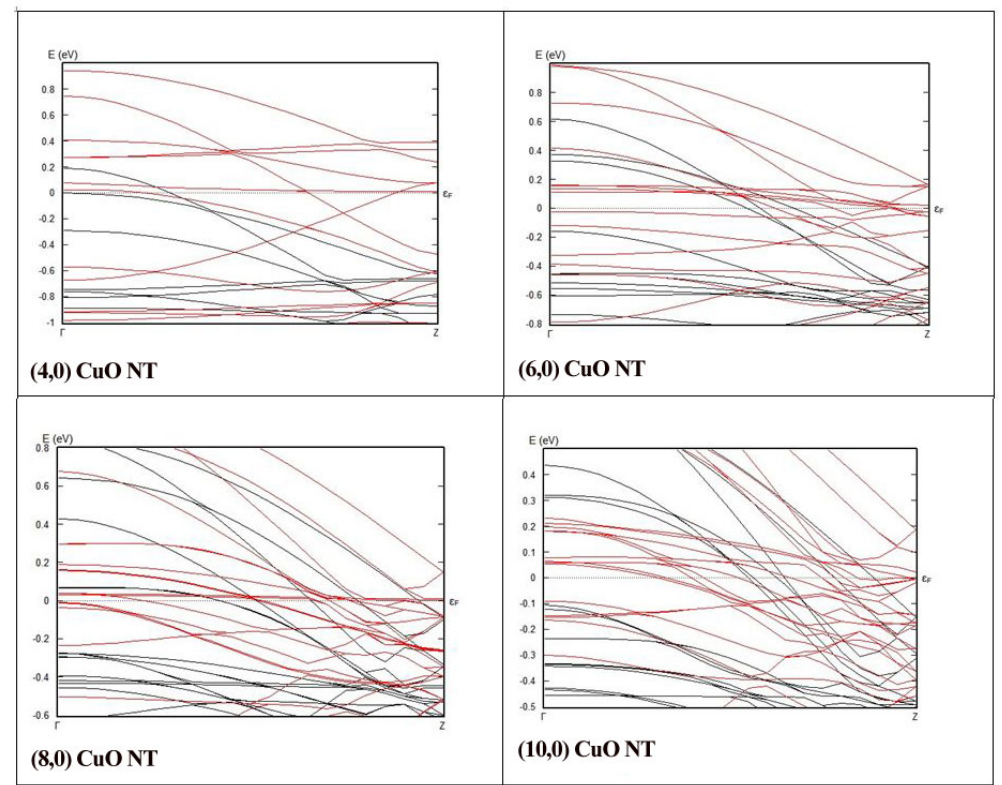

Figure 2: (color online) Band structure of CuO NTs with majority(black)) and minority (red)Spin

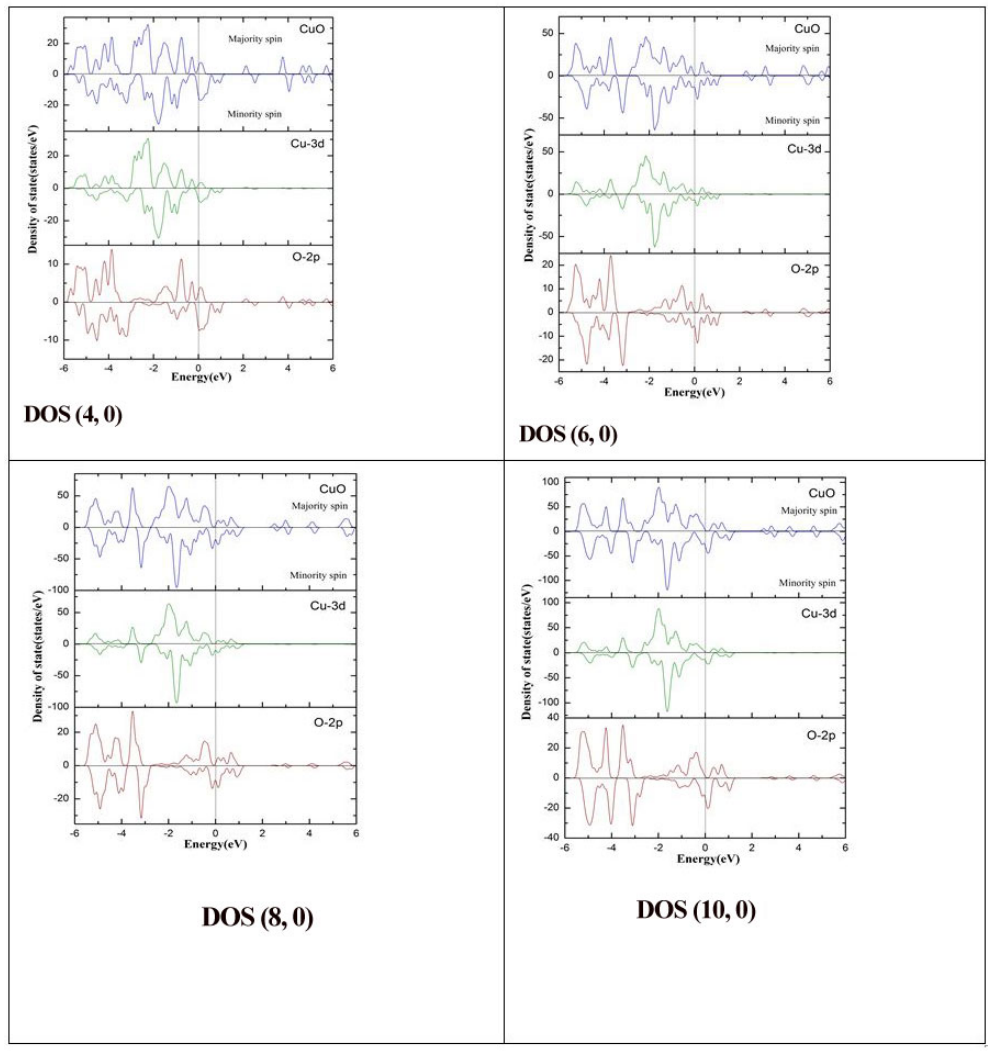

Figure 3: (color online) Spin Polarized DOS of CuO NTs at room temperature

\section{Magnetic moment}

It would be exciting to analyze the magnetic properties because of the metallic nature of $\mathrm{CuO}$ nanotubes. Magnetic moment of zigzag $\mathrm{CuO}$ nanotube at room temperature listed in Table 1 on which we observe that the magnetic moment is high for $(4,0)(1.95$ $\AA$ ), after increasing in diameter magnetic moment is decreases but at for $(10,0)(1.90 \AA)$ structure, it starts increases (Figure 4$)$. The 
magnetic moment of bulk $\mathrm{CuO}$ is $0.86 \mu \mathrm{B}$ /atom and individual $\mathrm{Cu}, \mathrm{O}$ atoms values are $0.68 \mu \mathrm{B} /$ atom and $0.18 \mu \mathrm{B} /$ atom respectively [8]. Magnetic moment is induced due to the exchange-splitting between states, here exchange splitting of the Cu-3d and $\mathrm{O}-2 \mathrm{p}$ states provide magnetic moment to $\mathrm{CuO}$ NTs. The fluctuation on magnetic moment on increasing diameter are due to increase in number and decrease in bond length between $\mathrm{Cu}$ and $\mathrm{O}$ atom. According to Hund's rule of magnetism if the bond length is decrease and number of atom increased that means the atom coming together and interaction between them increases, here this is responsible for decreasing magnetic moment [24]. The total magnetic moment $\mu_{\text {total }}(\mu \mathrm{B} /$ atom) of CuO nanotubes are linked directly to the individual magnetic moment of $\mathrm{Cu}$ and $\mathrm{O}$ atoms, strong interaction decrease their individual magnetic moment and affect the total magnetic moment of $\mathrm{CuO}$ nanotubes.

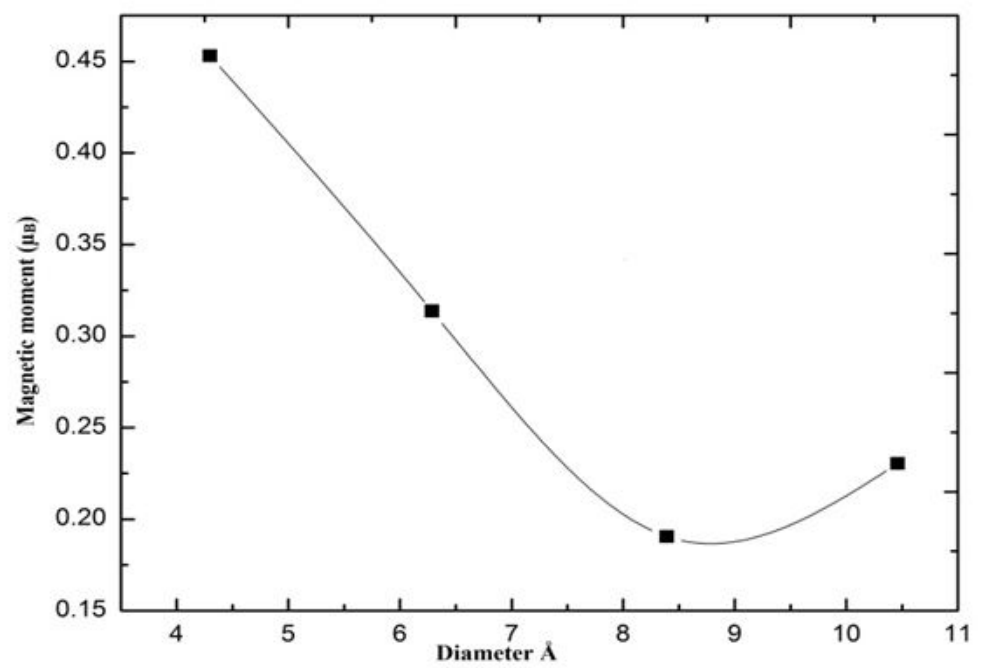

Figure 4: (color online) Magnetic moment as a function of diameter at room temperature.

Further we analyzed magnetic moment with different temperature. The temperature variation of the nano tubes is performed manually; it actually represents the temperature under which calculations are performed. We found that magnetic moment have high value at room temperature $(300 \mathrm{~K})$ then decrease with increasing temperature which is shown at Figure 5 . But for $(8,0)$ NT magnetic moment is increasing with increasing temperature. In our result maximum magnetic moment is observed at $4.30 \AA[$ [ $(4,0)$ $\mathrm{NT}]$ and minimum at $8.39 \AA[(8,0) \mathrm{NT}]$.

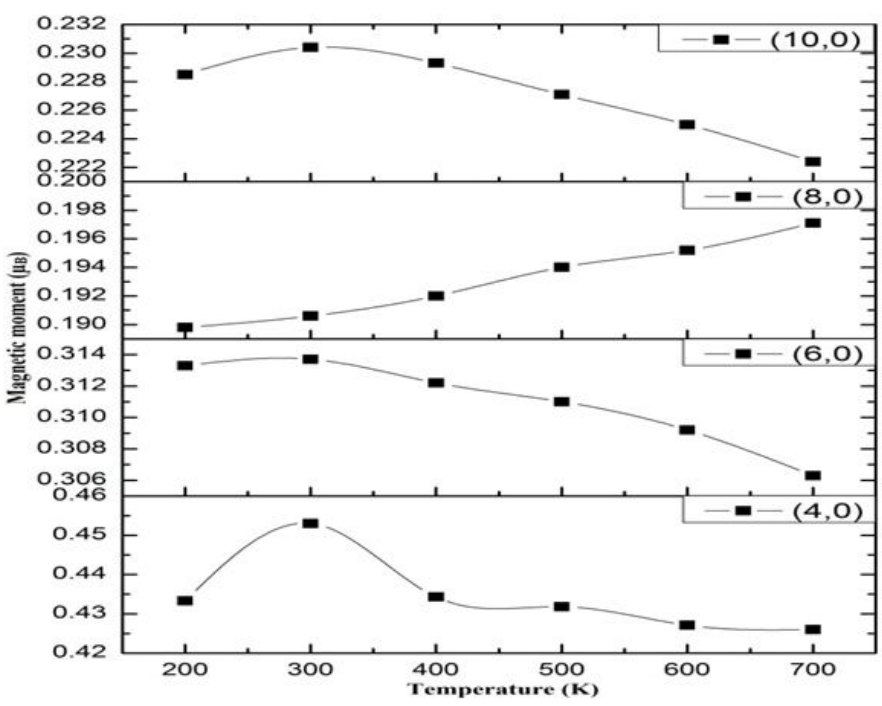

Figure 5: (color online) Magnetic moment as function of Temperature (K).

\section{Conclusion}

Structural, electronic and magnetic properties of zigzag nano tube of $(4,0),(6,0),(8,0)$ and $(10,0)$ NT were analyzed by density function theory based on ab-initio code ATK-VNL. Buckling has been occurred on optimized geometry structure. The magnitude of buckling is decreases on increasing diameter of tube. Furthermore as diameter increases the number of atoms are also increases, however binding energy of $\mathrm{CuO}$ nano tube increases at first and tends towards the saturation with increasing number of atoms. The hybridization of $\mathrm{Cu} 3 \mathrm{p}$ state and $\mathrm{O} 2 \mathrm{p}$ state make $\mathrm{CuO}$ nanotube metallic. Magnetic moments were calculated through Mulliken population analysis of $\mathrm{CuO}$ nanotube. Magnetic moments with respect to different diameter and different temperature were investigated. Highest value of magnetic moment $0.453 \mu \mathrm{B}$ was observed for $(4,0)$ at $300 \mathrm{~K}$, which continuously decreases for higher 
diameter nanotubes which attains minimum value at $(10,0)$ beyond that it is found to be increased. We were also analyzed relation of temperature and magnetic moment. All study show peak value at room temperature except $(8,0)$. In $(8,0)$ nanotube magnetic moment is increase with temperature. As our findings are first in series, it would certainly be of great importance to the theoretical as well as experimental workers of this area. $\mathrm{CuO}$ show remarkable magnetic moment indicating that we can use it in spintronics devices, memory devices and magnetic sensors. We are seeking the experimental verification of these data. If verified CuO can be used as binary analog to other CNT with novel magnetic properties.

\section{Acknowledgment}

We are thankful to ABV-IIITM, Gwalior for providing the computational nano-Science and Technology lab for entire computational work. HRPC, Nepal is also acknowledged for partial support for this project.

\section{References}

1. Batlle X, Labarta AL (2002) Finite-size effects in fine particles: magnetic and transport properties. J Phys D Appl Phys 35: R15-42.

2. Miao J, Hu W, Guo N, Lu Z, Zou X, et al. (2014) Single InAs Nanowire Room-Temperature Near-Infrared Photodetectors. ACS Nano 8: 3628-35.

3. Mattheiss LF (1972) Electronic Structure of the 3 d Transition-Metal Monoxides. I. Energy-Band Results. Phys Rev B 5: 290-306.

4. Harrison WA (2007) Heisenberg exchange in the magnetic monoxides. Phys Rev B 76: 10.1103/PhysRevB.76.054417.

5. Anisimov VI, Zaanen J, Andersen OK (1991) Band theory and Mott insulators: Hubbard U instead of Stoner I. Phys Rev B Condens Matter 44: 943-54.

6. Bednorz JG, Müller KA (1988) Perovskite-Type Oxides—-the New Approach to High-Tc Superconductivity. Nobel Lecture. Angewandte Chemie 27: 735-48.

7. Bednorz JG, Müller KA (1986) Possible high Tc superconductivity in the Ba- La- Cu- O system. Z Phys Rev B Condens Matter 64: 189-93.

8. Ekuma CE, Anisimov VI, Moreno J, Jarrell M (2014) Electronic structure and spectra of CuO. Eur Phys J B 87: 10.1140/epjb/e2013-40949-5.

9. Paudel S, Dandeliya S, Chaurasiya R, Srivastava A, Kaphle GC (2016). Magnetism in zigzag and armchair CuO nanotubes: Ab-initio study. J Magn Magn Mater 406: 8-14.

10. Wu D, Zhang Q, Tao M (2006) LSDA+ U study of cupric oxide: Electronic structure and native point defects. Phys Rev B 73: 10.1103/PhysRevB.73.235206.

11.Wang W, Liu Z, Liu Y, Xu C, Zheng C, et al. (2003) A simple wet-chemical synthesis and characterization of CuO nanorods. Appl Phys A 76: 417-20.

12. Kohn W, Sham LJ (1965) Self-consistent equations including exchange and correlation effects. Phys Rev B 140: A1133-8.

13. Dreizler RM, Gross EKU (1990) Density Functional Theory: An Approach to the Quantum Many-Body Problem, Springer Science \& Business Media, Germany.

14. Quantum Wise (2014) Atomistix ToolKit version 11.8.2 and 2014.2 QuantumWise, Denmark.

15. Brandbyge M, Mozos JL, Ordejón P, Taylor J, Stokbro K (2002) Density-functional method for nonequilibrium electron transport. Phys Rev B Condens Matter 65: 10.1103/PhysRevB.65.165401.

16. Stokbro K, Taylor J, Brandbyge M, Ordejón P (2003) TranSIESTA: a spice for molecular electronics. Ann N Y Acad Sci 1006: 212-26.

17. Taylor J, Guo H, Wang J (2001) Ab initio modeling of quantum transport properties of molecular electronic devices. Physical Review B 63: 10.1103/PhysRevB.63.245407.

18. Soler J M, Artacho E, Gale JD, García A, Junquera J, et al. (2002) The SIESTA method for ab initio order-N materials simulation. Phys Rev B Condens Matter 14: 2745-79.

19. Wu Y, Lai Y, Zhang ZQ (2011) Elastic metamaterials with simultaneously negative effective shear modulus and mass density. Phys Rev Lett $107: 105506$.

20. Perdew JP, Burke K, Ernzerhof M (1996) Generalized gradient approximation made simple. Phys Rev Lett 77: 3865-8.

21. Bachelet GB, Hamann DR, Schlüter M (1982) Pseudopotentials that work: From H to Pu. Phys Rev B 26: 4199-228.

22. Farrell HH, Parra RD (2011) Oxide nanotube analogues: CuO nanobarrels. J Vac Sci Technol B Nanotechnol Microelectron 29: 10.1116/1.3661990.

23. Luo LB, Wang XH, Xie C, Li ZJ, Lu R, et al. (2014) One-dimensional CuO nanowire: synthesis, electrical, and optoelectronic devices application. Nanoscale Res Lett 9: 1-8.

24. Bachelet GB, Hamann DR, Schlüter M (1982) Pseudopotentials that work: From H to Pu. Phys Rev B 26: 4199-228.

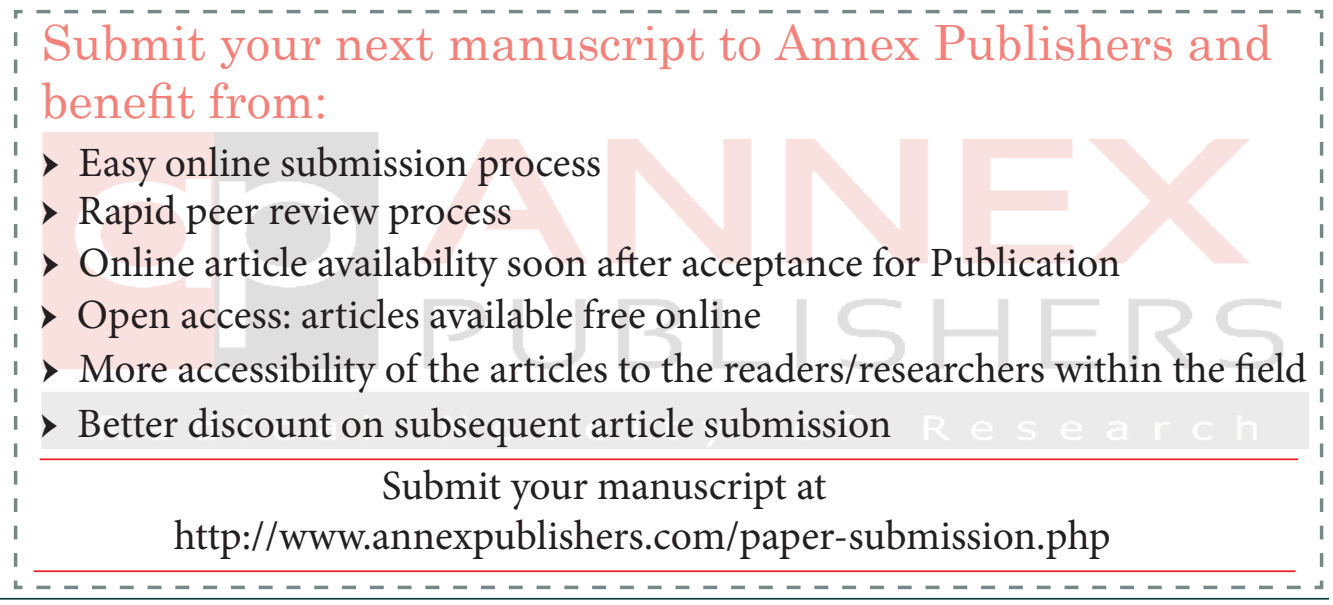

